\title{
ESTRUTURA DO ESTRATO ARBÓREO E REGENERATIVO DE UM FRAGMENTO DE FLORESTA ESTACIONAL SEMIDECIDUAL EM ITUMBIARA, GO
}

\section{STRUCTURE OF THE TREE AND REGENERATION LAYER ON A SEASONAL SEMIDECIDUOUS FOREST FRAGMENT IN ITUMBIARA, GOIÁS STATE}

\author{
Michel Eduardo Valentim Milhomem ${ }^{1}$ Glein Monteiro Araújo ${ }^{2}$ Vagner Santiago do Vale ${ }^{3}$
}

\section{RESUMO}

As Florestas Estacionais Semideciduais ocorrem em alguns locais no Bioma Cerrado, mas estão sendo suprimidas em meio à ampla matriz agropecuária no Brasil Central. O objetivo do trabalho foi caracterizar a estrutura horizontal e vertical, as síndromes de dispersão, os grupos sucessionais e a similaridade florística do estrato arbóreo e regenerativo de um trecho de fragmento de Floresta Estacional Semidecidual no município de Itumbiara, GO. Todos os indivíduos arbóreos vivos com circunferência do tronco a altura do peito $(\mathrm{CAP}) \geq 15$ foram identificados e medidos quanto à altura e circunferência do tronco em 25 parcelas de $20 \times 20 \mathrm{~m}$. A amostragem do estrato regenerativo foi realizada em 25 subparcelas de $10 \mathrm{~m} \times 10 \mathrm{~m}$, totalizando $0,25 \mathrm{ha}$, alocadas no vértice inferior direito das parcelas utilizadas para a amostragem do estrato arbóreo. Nestas subparcelas foram identificados e contados todos os indivíduos regenerantes de espécies arbóreas com altura maior ou igual a $1 \mathrm{~m}$ até aqueles com CAP $<15 \mathrm{~cm}$. Para o estrato arbóreo foi calculados: a densidade, frequência, dominância, valor de importância e a estratificação vertical. Nos dois estratos foram amostradas 100 espécies. A espécie com maior valor de importância no estrato arbóreo foi Nectandra megapotamica (Spreng) Mez, e no estrato regenerativo Siparuna guianensis Aubl teve maior número de indivíduos. Ambos os estratos apresentaram maior proporção de espécies secundárias iniciais e a zoocoria foi a síndrome de dispersão mais frequente. A estrutura fitossociológica atual mostra que o fragmento contém diversas espécies pouco representadas em outras Florestas Semideciduais, o que mostra a sua importância como área de preservação permanente.

Palavras-chave: estratificação; grupos ecológicos; síndrome de dispersão.

\begin{abstract}
Semideciduous forests occur in a few places in the Cerrado biome, but are being suppressed through by agriculture and livestock in central Brazil. The objective of this study was characterize the horizontal and vertical structures, the dispersal syndromes, successional groups and floristic similarity of the tree layer and regeneration in a semideciduous forest fragment in Itumbiara, Goiás state. All trees alive with circumference at breast height $(\mathrm{CAP}) \geq 15$ were identified and measured for height and diameter in 25 plots of $20 \times 20 \mathrm{~m}$. To the regenerative stratum the criteria was the inclusion of individuals with height $\geq 1 \mathrm{~m}$ until $\mathrm{CAP}=14.9 \mathrm{~cm}$ in 25 plots of $10 \times 10 \mathrm{~m}$. In both layers were sampled 100 species. The species with the highest importance in the tree layer were Nectandra megapotamica (Spreng.) Mez, and in the regenerative stratum, Siparuna guianensis Aubl. Both layers had a higher proportion of early secondary species and the zoochory dispersion syndrome was more frequent. Several important species are not represented in other semideciduous forests, and then this forest is an important permanent conservation area.
\end{abstract}

Keywords: stratification; ecological groups; dispersion syndrome.

1 Biólogo, Msc., Ecologia e Conservação de Recursos Naturais, Laboratório de Ecologia Vegetal, Universidade Federal de Uberlândia, Rua Ceará, s/n, Bloco 2D, sala 26, Umuarama, CEP 38400-902, Uberlândia (MG), Brasil. michelmilhomem@hotmail.com

2 Biólogo, Dr., Professor Adjunto do Instituto de Biologia, Laboratório de Ecologia Vegetal, Universidade Federal de Uberlândia, Rua Ceará, s/n, Bloco 2D, sala 26, Umuarama, CEP 38400-902, Uberlândia (MG), Brasil. glein@ufu.br

3 Biólogo, Dr., Bolsista CAPES/PNPD, Programa de Pós-Graduação em Ecologia e Conservação de Recursos Naturais, Laboratório de Ecologia Vegetal, Universidade Federal de Uberlândia, Rua Ceará, s/n, Bloco 2D, sala 26, Umuarama, CEP 38400-902, Uberlândia (MG), Brasil. vsvale@hotmail.com

Recebido para publicação em 08/02/2012 e aceito em 13/08/2012 


\section{INTRODUÇÃO}

Dentre as fitofisionomias da Floresta Atlântica, há uma diferenciação ditada pela sazonalidade de chuvas, separando as florestas não estacionais, sem demarcado período seco durante o ano, daquelas florestas cuja pluviosidade apresenta demarcada estação chuvosa e seca (VELOSO, 1991; OLIVEIRA-FILHO e FONTES, 2000). No Brasil Central, estas florestas são encontradas em forma de manchas florestais em meio à vegetação aberta, principalmente na região do Cerrado (RIZZINI, 1997) e são chamadas por Veloso et al. (1991) de Florestas Estacionais Semideciduais (FES). Pelo fato das FES estarem localizadas em biomas considerados "hotspots" para a conservação da biodiversidade mundial (Floresta Atlântica e Cerrado) (MYERS et al., 2000), merecem especial atenção visando à manutenção de sua diversidade vegetal.

Apesar das FES, do Bioma Cerrado, possuírem solos ácidos e distróficos, principalmente em camadas mais profundas (ARAÚJO e HARIDASAN, 1997), muitas FES foram suprimidas devido à expansão da fronteira agrícola (TURNER, 1996). Nas regiões mais degradadas, estas fisionomias restringem-se a pequenos fragmentos, próximos a nascentes ou cursos d'água (ARAÚJO e HARIDASAN, 1997). Tais fragmentos florestais merecem atenção especial por estarem, geralmente, em ambientes em diferentes fases de regeneração, com marcantes efeitos de borda (NUNES et al., 2003) e por serem importantes objetos de estudo que auxiliam no entendimento de questões relacionadas à automanutenção dessas florestas em uma matriz antropizada.

Diversos trabalhos têm buscado aprofundar os conhecimentos do componente arbóreo a fim de oferecer mais informações sobre o estágio de conservação destas florestas. Em São Paulo, alguns trabalhos apontam a grande diversidade de polinizadores e dispersores dependentes da comunidade arbórea (YAMAMOTO et al., 2005; KINOSHITA et al., 2006). No Sul de Minas Gerais, Machado et al. (2004), Silva et al. (2004) e Toniato e Oliveira-Filho (2004) demonstraram a importância de diferentes grupos sucessionais de plantas na estrutura dessas comunidades florestais. No Triângulo Mineiro, a manutenção da matriz de entorno e a ausência de antropização no interior de alguns fragmentos de FES, permitem dominância de espécies secundárias tardias tolerantes à sombra no sub-bosque (DIAS NETO et al., 2009; VALE et al., 2009). Assim, tais trabalhos têm acrescido informações importantes capazes de aprimorar os estudos básicos da estrutura e síndrome de dispersão de espécies arbóreas das FES.

Outro aspecto fundamental, porém, ainda pouco abordado, é a estrutura do estrato regenerativo de espécies arbóreas (SOUZA et al., 2006; VIEIRA e SCARIOT, 2006; SALLES e SCHIAVINI, 2007; MARANGON et al., 2008). Diferente do componente arbóreo, este estrato representa indivíduos jovens da comunidade, muitas vezes mais vulneráveis a impactos antrópicos que modificam as condições ambientais das florestas, como a umidade e a luminosidade, por exemplo. Por isso, é necessário compreender os processos de regeneração natural em florestas tropicais, buscando prever a provável estrutura e composição da floresta no futuro (PARE et al., 2009; CECCON et al., 2006).

Apesar da importância da estrutura vertical, a maioria das investigações realizadas em Florestas Semideciduais enfatiza somente a estrutura horizontal, simplificando a comunidade (VALE et al., 2009). Alguns estudos realizados em Florestas Estacionais têm demonstrado diferenças entre o dossel formado, principalmente, por espécies tardias de grande porte e sub-bosque formado por espécies tardias de pequeno porte, pouco eficientes em sobreviver em grandes clareiras (PAULA et al., 2004; VALE et al., 2009).

Uma vez compreendida a estrutura da comunidade, é possível, com auxílio do conhecimento das características das espécies quanto ao seu requerimento de luz, inferir sobre o estágio sucessional do fragmento (SWAINE e WHITMORE, 1988). Para isso, as espécies são classificadas em grupos sucessionais, que representam uma ferramenta importante para se avaliar a comunidade arbórea em fragmentos florestais (OLIVEIRA-FILHO et al., 1997; TABARELLI e MANTOVANI, 1999; NUNES et al., 2003). Desta forma, o presente trabalho teve como objetivo caracterizar a estrutura horizontal e vertical, as síndromes de dispersão e os grupos sucessionais do estrato arbóreo e regenerativo de um trecho de fragmento de Floresta Estacional Semidecidual no município de Itumbiara, GO.

\section{MATERIAL E MÉTODOS}

O trabalho foi realizado em um trecho de fragmento $\left(18^{\circ} 20^{\prime} 54^{\prime \prime}\right.$ 'S e $\left.49^{\circ} 04^{\prime} 26^{\prime \prime} \mathrm{W}\right)$ de Floresta Estacional Semidecidual (VELOSO et al., 1991) localizado no município de Itumbiara, GO, inserido em uma matriz de cultivo de cana-de-açúcar 
nas margens da represa da Usina Hidroelétrica de Itumbiara. O fragmento, de aproximadamente 60 ha, trata-se da reserva legal de uma propriedade particular. Este apresenta diferentes níveis de antropização, resultante de corte seletivo de espécies arbóreas ocorridas a cerca de 20 anos. Este fragmento é um remanescente de uma área florestal mais ampla que ocupava as margens do rio Paranaíba no município de Itumbiara. Atualmente, o fragmento está protegido do corte de madeira, queimadas e caça e tem em suas proximidades, fragmentos menores da mesma fisionomia vegetal. O clima da região é a Aw (KÖPPEN, 1948) caracterizado por verões chuvosos e invernos secos. A precipitação anual total é de $1500 \mathrm{~mm}$ de chuva ocorrendo, principalmente, de outubro a março, sendo os demais meses relativamente secos, quando o déficit hídrico é acentuado (MARCHIORI et al., 2003). Na região, onde se encontra o fragmento, são encontrados, principalmente, Latossolos Vermelhos Distróficos, de acordo com a classificação do IBGE (2001).

O estudo fitossociológico do estrato arbóreo foi realizado pelo método de parcelas (MUELLERDOMBOIS e ELLENBERG, 1974). Foram plotadas 25 parcelas de $20 \times 20 \mathrm{~m}$, dispostas em cinco transecções, distantes $10 \mathrm{~m}$ uma da outra, compostas por cinco parcelas contiguas, totalizando 1 ha. As parcelas situaram-se a cerca de $5 \mathrm{~m}$ da borda em um trecho mais preservado do fragmento. Nessas parcelas, todos os indivíduos arbóreos vivos com circunferência a $1,30 \mathrm{~m}$ do solo $(\mathrm{CAP}) \geq 15 \mathrm{~cm}$ foram identificados e tiveram medidas a sua circunferência e altura. A circunferência foi medida com auxílio de uma fita métrica e a altura foi determinada tendo como referência uma vara com $5 \mathrm{~m}$, sendo que indivíduos com altura superior a $5 \mathrm{~m}$ tiveram sua altura estimada visualmente. A avaliação do estrato regenerativo foi realizada em 25 subparcelas de $10 \mathrm{~m} \times 10 \mathrm{~m}$, totalizando $0,25 \mathrm{ha}$, alocadas no vértice inferior direito das parcelas utilizadas para a amostragem do estrato arbóreo. Nestas subparcelas foram identificadas as espécies e contados todos os indivíduos regenerantes de espécies arbóreas com altura maior ou igual a $1 \mathrm{~m}$ até aqueles com $\mathrm{CAP}<$ $15 \mathrm{~cm}$. Os indivíduos foram identificados no próprio local ou coletados para consultas no Herbário da Universidade Federal de Uberlândia (HUFU) e botânicos especialistas. O sistema de classificação de famílias, gêneros e espécies seguiu o sistema APG III (APG III, 2009). Para as espécies arbóreas com CAP $>15 \mathrm{~cm}$ foram calculados os seguintes parâmetros fitossociológicos: DR (densidade rela- tiva), DoR (dominância relativa), FR (frequência relativa) e VI (valor de importância). O valor de importância foi relativizado para porcentagem. Todos estes parâmetros foram calculados pelo programa FITOPAC 1.6.4.29 (SHEPHERD, 2006). Para estimar a riqueza da comunidade arbórea e do estrato regenerativo, foi calculado o estimador de riqueza Jackknife de segunda ordem por meio do programa Estimates version 7.5 (COWELL, 2005). Este estimador foi considerado por Cowell e Coddington (1994), em trabalho comparando estimadores de riqueza, como um bom estimador de riqueza em comparação a outros estimadores. Para avaliar a suficiência amostral dos estratos de regeneração e arbóreo, o mesmo estimador de riqueza foi utilizado (Jackknife de segunda ordem) por ser considerado um bom estimador para ser utilizado na construção da curva do coletor (KREBS, 2000).

A determinação da estrutura vertical das espécies arbóreas foi realizada pela metodologia descrita por Vale et al. (2009). Esta metodologia classifica a estrutura vertical em três estratos (Estrato 1 = Sub-bosque, Estrato 2 = Estrato intermediário e Estrato 3 = Dossel). Neste trabalho foram determinados os estratos apenas das espécies que apresentaram, no mínimo, 10 indivíduos amostrados. Os critérios e intervalos para classificação da estratificação vegetal são: Estrato 1 (denominado sub-bosque): Q3e $\leq \mathrm{Mc}$; Estrato 2 (denominado estrato intermediário): $\mathrm{Mc}<\mathrm{Q} 3 \mathrm{e} \leq \mathrm{Q} 3 \mathrm{c}$; Estrato 3 (denominado dossel): Q3e $>$ Q3c, Onde: $\mathrm{Q} 3 \mathrm{e}=$ terceiro quartil das alturas dos indivíduos amostrados da espécie; $\mathrm{Mc}=$ mediana das alturas dos indivíduos amostrados da comunidade; Q3c = terceiro quartil das alturas dos indivíduos (VALE et al., 2009).

As espécies também foram agrupadas de acordo com seus grupos sucessionais, segundo Gandolfi et al. (1995), em: pioneiras (Pi) - dependentes de luz que não ocorrem no sub-bosque, desenvolvendo-se em clareiras ou nas bordas da floresta; secundárias iniciais $(\mathrm{Si})$ - ocorrem em condições de sombreamento médio ou luminosidade não muito intensa, ocorrendo em clareiras pequenas, bordas de clareiras grandes e bordas de floresta; secundárias tardias $(\mathrm{St})$ - desenvolve-se no sub-bosque em condições de sombra leve ou densa e podem crescer até alcançar o dossel ou a condição de emergente.

Quanto às síndromes de dispersão, as espécies foram classificadas em: anemocóricas, zoocóricas e autocóricas. Tal classificação foi realizada por meio de consulta a artigos (ZIPPARRO et al., 2005; CATHARINO et al., 2006; PIVELLO et al., 2006; 
YAMAMOTO et al., 2007) e observações de campo, com base na classificação proposta por Van der Pijl (1982).

\section{RESULTADOS E DISCUSSÃO}

No estrato arbóreo foram amostrados, em um hectare, 1.116 indivíduos distribuídos em 77 espécies, 63 gêneros e 37 famílias (Tabela 1). Em comparação a outros estudos realizados em Florestas Estacionais Semideciduais, como as dos estudos de Dias Neto et al. (2009), Vale et al. (2009), Prado Júnior et al. (2010) no Triângulo
Mineiro, o número de espécie é semelhante ao atual estudo (70 a 90 espécies). Já a riqueza do estrato regenerativo foi de 68 espécies, inclusive com muitas espécies diferentes daquelas amostradas no estrato arbóreo. Assim, considerando a riqueza conjunta dos dois estratos, arbóreo e arbustivo, foram encontradas 100 espécies. A inclusão do estrato arbóreo regenerativo ocasionou um acréscimo de $23,0 \%$ de espécies, ilustrando que, quando não avaliado, o estrato regenerativo, a riqueza da floresta pode estar subestimada, independente do tipo de amostragem. A Figura 1 demonstra que a suficiência amostral foi mais próxima de ser atingida para o estrato arbóreo,

TABELA 1: Fitossociologia e características ecológicas, em ordem de Valor de Importância do estrato arbóreo (VI) das espécies arbóreas de um trecho de fragmento de Floresta Estacional Semidecidual em Itumbiara, GO.

TABLE 1: Species phytossociology and ecological characteristics, in order of arboreal importance value to a seasonal semideciduous forest in Itumbiara, Goiás.

\begin{tabular}{|c|c|c|c|c|c|c|c|c|c|}
\hline Espécie & NA & NR & DR & DoR & FR & VI & GS & SD & EV \\
\hline Nectandra megapotamica (Spreng.) Mez & 239 & 308 & 21,42 & 20,08 & 5,46 & 15,65 & $\mathrm{Si}$ & Zo & Do \\
\hline Schefflera morototoni (Aubl.) Maguire, Steyerm. \& Frodin & 58 & 24 & 5,20 & 8,44 & 3,71 & 5,78 & $\mathrm{Si}$ & Zo & Do \\
\hline Aspidosperma discolor A.DC. & 38 & 220 & 3,41 & 9,66 & 4,15 & 5,74 & $\mathrm{Si}$ & An & Do \\
\hline Hirtella gracilipes (Hook.f.) Prance & 99 & 260 & 8,87 & 2,78 & 5,02 & 5,56 & $\mathrm{Si}$ & Zo & $\mathrm{Sb}$ \\
\hline Protium heptaphyllum (Aubl.) Marchand & 68 & 208 & 6,09 & 4,43 & 5,02 & 5,18 & $\mathrm{Si}$ & Zo & In \\
\hline Callisthene major Mart. & 25 & 4 & 2,24 & 9,74 & 3,06 & 5,01 & $\mathrm{Si}$ & An & Do \\
\hline Virola sebifera Aubl. & 55 & 152 & 4,93 & 4,19 & 4,37 & 4,49 & $\mathrm{Si}$ & Zo & Do \\
\hline Emmotum nitens (Benth.) Miers & 57 & 100 & 5,11 & 3,18 & 4,80 & 4,36 & St & Zo & In \\
\hline Siparuna guianensis Aubl. & 65 & 780 & 5,82 & 0,77 & 4,59 & 3,73 & $\mathrm{Si}$ & Zo & $\mathrm{Sb}$ \\
\hline Ocotea corymbosa (Meisn.) Mez & 28 & 316 & 2,51 & 4,51 & 3,49 & 3,50 & $\mathrm{Si}$ & Zo & Do \\
\hline Xylopia aromatica (Lam.) Mart. & 41 & 96 & 3,67 & 1,74 & 4,59 & 3,33 & $\mathrm{Pi}$ & Zo & In \\
\hline Maprounea guianensis Aubl. & 33 & 8 & 2,96 & 2,61 & 3,49 & 3,02 & $\mathrm{Si}$ & Zo & In \\
\hline Annona montana Macfad. & 36 & 96 & 3,23 & 2,70 & 3,06 & 3,00 & $\mathrm{Si}$ & Zo & In \\
\hline Cheiloclinium cognatum (Miers) A.C.Sm. & 37 & 672 & 3,32 & 0,63 & 3,49 & 2,48 & St & Zo & $\mathrm{Sb}$ \\
\hline Micropholis venulosa (Mart. \& Eichler) Pierre & 9 & 40 & 0,81 & 4,31 & 1,31 & 2,14 & St & Zo & $\mathrm{c}$ \\
\hline Siphoneugena densiflora O.Berg & 21 & 116 & 1,88 & 1,07 & 2,84 & 1,93 & St & Zo & In \\
\hline Licania apetala (E.Mey.) Fritsch & 11 & 104 & 0,99 & 2,38 & 2,18 & 1,85 & $\mathrm{Si}$ & Zo & In \\
\hline Antonia ovata Pohl & 18 & 32 & 1,61 & 1,39 & 2,18 & 1,73 & $\mathrm{Si}$ & Zo & In \\
\hline Terminalia glabrescens Mart. & 16 & 20 & 1,43 & 0,94 & 1,97 & 1,45 & St & An & In \\
\hline Vochysia haenkeana Mart. & 11 & 36 & 0,99 & 0,61 & 2,18 & 1,26 & $\mathrm{Si}$ & An & In \\
\hline Hymenaea courbaril $\mathrm{L}$. & 4 & 4 & 0,36 & 1,59 & 0,87 & 0,94 & St & Zo & $\mathrm{c}$ \\
\hline Dipteryx alata Vogel & 3 & $\mathrm{~b}$ & 0,27 & 1,43 & 0,66 & 0,79 & $\mathrm{Si}$ & Zo & $\mathrm{c}$ \\
\hline Hirtella glandulosa Spreng. & 4 & $\mathrm{~b}$ & 0,36 & 1,06 & 0,87 & 0,77 & $\mathrm{Si}$ & Zo & $\mathrm{c}$ \\
\hline Licania humilis Cham. \& Schltdl. & 6 & $\mathrm{~b}$ & 0,54 & 0,42 & 1,31 & 0,76 & $\mathrm{Si}$ & Zo & $\mathrm{c}$ \\
\hline Styrax camporum Pohl & 9 & $\mathrm{~b}$ & 0,81 & 0,11 & 1,31 & 0,74 & $\mathrm{Si}$ & Zo & $\mathrm{c}$ \\
\hline Ormosia arborea (Vell.) Harms & 7 & 4 & 0,63 & 0,17 & 1,31 & 0,70 & St & $\mathrm{Au}$ & $\mathrm{c}$ \\
\hline Roupala montana Aubl. & 4 & 28 & 0,36 & 0,79 & 0,87 & 0,68 & St & An & $\mathrm{c}$ \\
\hline Ocotea minarum (Nees \& C.Mart.) Mez & 5 & $\mathrm{~b}$ & 0,45 & 0,41 & 1,09 & 0,65 & St & Zo & $\mathrm{c}$ \\
\hline Myrcia tomentosa (Aubl.) DC. & 5 & 12 & 0,45 & 0,18 & 1,09 & 0,57 & $\mathrm{Si}$ & Zo & $\mathrm{c}$ \\
\hline
\end{tabular}


TABELA 1: Continuação ...

TABLE 1: Continued ...

\begin{tabular}{|c|c|c|c|c|c|c|c|c|c|}
\hline Espécie & NA & NR & DR & DoR & FR & VI & GS & SD & EV \\
\hline Tapirira guianensis Aubl. & 4 & 4 & 0,36 & 0,36 & 0,87 & 0,53 & $\mathrm{Si}$ & Zo & $\mathrm{c}$ \\
\hline Vatairea macrocarpa (Benth.) Ducke & 4 & $\mathrm{~b}$ & 0,36 & 0,55 & 0,66 & 0,52 & St & An & $\mathrm{c}$ \\
\hline Licania kunthiana Hook.f. & 3 & 120 & 0,27 & 0,59 & 0,66 & 0,51 & $\mathrm{Si}$ & Zo & $\mathrm{c}$ \\
\hline Qualea dichotoma (Mart.) Warm. & 4 & $\mathrm{~b}$ & 0,36 & 0,25 & 0,87 & 0,49 & St & An & $\mathrm{c}$ \\
\hline Campomanesia velutina (Cambess.) O.Berg & 5 & $\mathrm{~b}$ & 0,45 & 0,07 & 0,87 & 0,46 & $\mathrm{Si}$ & Zo & $\mathrm{c}$ \\
\hline Senegalia polyphylla (DC) Britton \& Rose & 5 & $\mathrm{~b}$ & 0,45 & 0,27 & 0,66 & 0,46 & $\mathrm{Pi}$ & $\mathrm{Au}$ & $\mathrm{c}$ \\
\hline Agonandra brasiliensis Miers ex Benth. \& Hook.f. & 4 & $\mathrm{~b}$ & 0,36 & 0,10 & 0,87 & 0,44 & St & Zo & $\mathrm{c}$ \\
\hline Inga laurina $(\mathrm{Sw}$.$) Willd.$ & 4 & 4 & 0,36 & 0,06 & 0,87 & 0,43 & $\mathrm{Si}$ & Zo & $\mathrm{c}$ \\
\hline Cardiopetalum calophyllum Schltdl. & 4 & 48 & 0,36 & 0,05 & 0,87 & 0,43 & $\mathrm{Si}$ & Zo & $\mathrm{c}$ \\
\hline Pouteria torta (Mart.) Radlk. & 1 & $\mathrm{~b}$ & 0,09 & 0,95 & 0,22 & 0,42 & St & Zo & $\mathrm{c}$ \\
\hline Pouteria gardneri (Mart. \& Miq.) Baehni & 4 & 8 & 0,36 & 0,23 & 0,66 & 0,42 & $\mathrm{Si}$ & Zo & $\mathrm{c}$ \\
\hline Luehea grandiflora Mart. & 3 & 4 & 0,27 & 0,18 & 0,66 & 0,37 & $\mathrm{Si}$ & An & $\mathrm{c}$ \\
\hline Nectandra membranacea (Sw.) Griseb. & 3 & 140 & 0,27 & 0,12 & 0,66 & 0,35 & St & Zo & $\mathrm{c}$ \\
\hline Eugenia florida DC. & 3 & 16 & 0,27 & 0,06 & 0,66 & 0,33 & $\mathrm{Si}$ & Zo & $\mathrm{c}$ \\
\hline Ixora brevifolia Benth. & 4 & 4 & 0,36 & 0,08 & 0,44 & 0,29 & St & Zo & $\mathrm{c}$ \\
\hline Astronium nelsonrosae Santin & 1 & $\mathrm{~b}$ & 0,09 & 0,54 & 0,22 & 0,28 & St & An & $\mathrm{c}$ \\
\hline Eugenia brasiliensis Lam. & 3 & $\mathrm{~b}$ & 0,27 & 0,14 & 0,44 & 0,28 & $\mathrm{Si}$ & Zo & $\mathrm{c}$ \\
\hline Margaritaria nobilis L.f. & 3 & $\mathrm{~b}$ & 0,27 & 0,14 & 0,44 & 0,28 & $\mathrm{Si}$ & Zo & $\mathrm{c}$ \\
\hline Qualea grandiflora Mart. & 2 & $\mathrm{~b}$ & 0,18 & 0,23 & 0,44 & 0,28 & St & An & $\mathrm{c}$ \\
\hline Platypodium elegans Vogel & 2 & $\mathrm{~b}$ & 0,18 & 0,22 & 0,44 & 0,28 & $\mathrm{Si}$ & An & $\mathrm{c}$ \\
\hline Copaifera lansdorffii Desf. & 1 & 20 & 0,09 & 0,48 & 0,22 & 0,26 & St & Zo & $\mathrm{c}$ \\
\hline Pera glabrata (Schott) Poepp. ex Baill. & 3 & $\mathrm{~b}$ & 0,27 & 0,05 & 0,44 & 0,25 & $\mathrm{Si}$ & Zo & $\mathrm{c}$ \\
\hline Guazuma ulmifolia Lam. & 2 & $\mathrm{~b}$ & 0,18 & 0,12 & 0,44 & 0,24 & $\mathrm{Pi}$ & Zo & $\mathrm{c}$ \\
\hline Coccoloba mollis Casar. & 2 & 4 & 0,18 & 0,05 & 0,44 & 0,22 & $\mathrm{Si}$ & Zo & $\mathrm{c}$ \\
\hline Diospyros hispida A.DC. & 2 & 4 & 0,18 & 0,04 & 0,44 & 0,22 & $\mathrm{Si}$ & Zo & $\mathrm{c}$ \\
\hline Matayba guianensis Aubl. & 2 & 52 & 0,18 & 0,03 & 0,44 & 0,22 & $\mathrm{Si}$ & Zo & $\mathrm{c}$ \\
\hline Casearia gossypiosperma Briq. & 3 & 40 & 0,27 & 0,14 & 0,22 & 0,21 & $\mathrm{Si}$ & An & $\mathrm{c}$ \\
\hline Machaerium stipitatum (DC.) Vogel & 1 & $\mathrm{~b}$ & 0,09 & 0,30 & 0,22 & 0,20 & $\mathrm{Si}$ & An & $\mathrm{c}$ \\
\hline Tachigali vulgaris L.G.Silva \& H.C.Lima. & 1 & 16 & 0,09 & 0,28 & 0,22 & 0,20 & $\mathrm{Pi}$ & An & $\mathrm{c}$ \\
\hline Lamanonia ternata Vell. & 1 & $\mathrm{~b}$ & 0,09 & 0,21 & 0,22 & 0,17 & $\mathrm{Si}$ & Zo & $\mathrm{c}$ \\
\hline Aspidosperma cylindrocarpon Müll.Arg. & 1 & 4 & 0,09 & 0,18 & 0,22 & 0,16 & St & An & $\mathrm{c}$ \\
\hline Plathymenia reticulata Benth. & 2 & $\mathrm{~b}$ & 0,18 & 0,06 & 0,22 & 0,15 & St & $\mathrm{Au}$ & $\mathrm{c}$ \\
\hline Byrsonima laxiflora Griseb. & 1 & $\mathrm{~b}$ & 0,09 & 0,12 & 0,22 & 0,14 & $\mathrm{Si}$ & Zo & $\mathrm{c}$ \\
\hline Lacistema aggregatum (P.J.Bergius) Rusby & 2 & $\mathrm{~b}$ & 0,18 & 0,02 & 0,22 & 0,14 & $\mathrm{Pi}$ & Zo & $\mathrm{c}$ \\
\hline Ficus trigona L.f. & 1 & $\mathrm{~b}$ & 0,09 & 0,08 & 0,22 & 0,13 & St & Zo & $\mathrm{c}$ \\
\hline Machaerium acutifolium Mart.ex Benth. & 1 & $\mathrm{~b}$ & 0,09 & 0,08 & 0,22 & 0,13 & $\mathrm{Si}$ & An & $\mathrm{c}$ \\
\hline Handroanthus chrysotrichus (Mart. ex A.DC.) Mattos & 1 & $\mathrm{~b}$ & 0,09 & 0,06 & 0,22 & 0,12 & $\mathrm{Si}$ & An & $\mathrm{c}$ \\
\hline Jacaranda cuspidifolia Mart. & 1 & $\mathrm{~b}$ & 0,09 & 0,03 & 0,22 & 0,11 & $\mathrm{Si}$ & An & $\mathrm{c}$ \\
\hline Myrcia splendens (Sw.) DC. & 1 & 40 & 0,09 & 0,03 & 0,22 & 0,11 & $\mathrm{Si}$ & Zo & $\mathrm{c}$ \\
\hline Apuleia leiocarpa (Vogel) J.F.Macbr. & 1 & $\mathrm{~b}$ & 0,09 & 0,02 & 0,22 & 0,11 & $\mathrm{Si}$ & An & $\mathrm{c}$ \\
\hline Cordia trichotoma (Vell.) Arráb. ex Steud. & 1 & $\mathrm{~b}$ & 0,09 & 0,03 & 0,22 & 0,11 & $\mathrm{Si}$ & An & $\mathrm{c}$ \\
\hline Erythroxylum subracemosum Turcz. & 1 & 4 & 0,09 & 0,02 & 0,22 & 0,11 & St & Zo & $\mathrm{c}$ \\
\hline Alibertia edulis (Rich.) A.Rich. ex DC. & 1 & $\mathrm{~b}$ & 0,09 & 0,01 & 0,22 & 0,11 & $\mathrm{Si}$ & Zo & $\mathrm{c}$ \\
\hline Cordiera sessilis (Vell.) Kuntze & 1 & 108 & 0,09 & 0,01 & 0,22 & 0,11 & $\mathrm{Pi}$ & Zo & $\mathrm{c}$ \\
\hline Maytenus aquifolia Mart. & 1 & $\mathrm{~b}$ & 0,09 & 0,01 & 0,22 & 0,11 & St & Zo & $\mathrm{c}$ \\
\hline Tabebuia roseoalba (Ridl.) Sandwith & 1 & 12 & 0,09 & 0,02 & 0,22 & 0,11 & St & An & $\mathrm{c}$ \\
\hline Tapirira obtusa (Benth.) J.D.Mitch. & 1 & 4 & 0,09 & 0,02 & 0,22 & 0,11 & $\mathrm{Si}$ & Zo & $\mathrm{c}$ \\
\hline
\end{tabular}


TABELA 1: Continuação ...

TABLE 1: Continued ...

\begin{tabular}{|c|c|c|c|c|c|c|c|c|c|}
\hline Espécie & NA & NR & DR & DoR & FR & VI & GS & SD & $\mathrm{EV}$ \\
\hline Zanthoxylum rhoifolium Lam. & 1 & $\mathrm{~b}$ & 0,09 & 0,02 & 0,22 & 0,11 & $\mathrm{Si}$ & Zo & $\mathrm{c}$ \\
\hline Coussarea hydrangeifolia (Benth.) \& Hook.f. & a & 144 & - & - & - & - & $\mathrm{Si}$ & Zo & - \\
\hline Astronium fraxinifolium Schott ex Spreng. & a & 44 & - & - & - & - & St & An & - \\
\hline Unonopsis lindmanii R.E.Fr. & a & 24 & - & - & - & - & St & Zo & - \\
\hline Miconia albicans (Sw.) Triana & $\mathrm{a}$ & 20 & - & - & - & - & $\mathrm{Pi}$ & Zo & - \\
\hline Sorocea bonplandii (Baill.) W.C.Burger et al. & $\mathrm{a}$ & 32 & - & - & - & - & $\mathrm{Si}$ & Zo & - \\
\hline Trichilia pallida $\mathrm{Sw}$ & a & 12 & - & - & - & - & $\mathrm{Si}$ & Zo & - \\
\hline Cupania vernalis Cambess. & a & 12 & - & - & - & - & St & Zo & - \\
\hline Ouratea castaneifolia (DC.) Engl. & a & 8 & - & - & - & - & $\mathrm{Si}$ & Zo & - \\
\hline Handroanthus serratifolius (Vahl) S.O.Grose & a & 8 & - & - & - & - & $\mathrm{Pi}$ & An & - \\
\hline Symplocos tetrandra Mart. ex Miq. & $\mathrm{a}$ & 4 & - & - & - & - & $\mathrm{Si}$ & Zo & - \\
\hline Pseudolmedia laevigata Trécul & $\mathrm{a}$ & 4 & - & - & - & - & St & Zo & - \\
\hline Erythroxylum daphnites Mart. & $\mathrm{a}$ & 4 & - & - & - & - & $\mathrm{Si}$ & Zo & - \\
\hline Inga sessilis (Vell.) Mart. & a & 8 & - & - & - & - & St & Zo & - \\
\hline Genipa americana $\mathrm{L}$. & $\mathrm{a}$ & 4 & - & - & - & - & $\mathrm{Si}$ & Zo & - \\
\hline Rudgea viburnoides (Cham.) Benth. & a & 4 & - & - & - & - & $\mathrm{Si}$ & Zo & - \\
\hline Garcinia brasiliensis Mart. & $\mathrm{a}$ & 4 & - & - & - & - & $\mathrm{Si}$ & Zo & - \\
\hline Simira viridiflora (Allemao \& Saldanha) Steyerm. & a & 4 & - & - & - & - & $\mathrm{Si}$ & An & - \\
\hline Trichilia elegans A.Juss. & $\mathrm{a}$ & 4 & - & - & - & - & St & Zo & - \\
\hline Rhamnidium elaeocarpum Reissek & a & 4 & - & - & - & - & $\mathrm{Si}$ & Zo & - \\
\hline Piptadenia gonoacantha (Mart.) J.F.Macbr. & $\mathrm{a}$ & 4 & - & - & - & - & $\mathrm{Pi}$ & $\mathrm{Au}$ & - \\
\hline Trichilia clausseni C.DC. & a & 4 & - & - & - & - & $\mathrm{Si}$ & Zo & - \\
\hline Sweetia fruticosa Spreng. & $\mathrm{a}$ & 4 & - & - & - & - & $\mathrm{Si}$ & An & - \\
\hline Chomelia bella (Standl.) Steyerm & $\mathrm{a}$ & 4 & - & - & - & - & $\mathrm{Si}$ & Zo & - \\
\hline
\end{tabular}

Em que: $\mathrm{NA}=$ número de indivíduos do estrato arbóreo (ind.ha $\left.{ }^{-1}\right) ; \mathrm{NR}=$ número de indivíduos presentes na regeneração (ind.ha ${ }^{-1}$ ); $\mathrm{DR}=$ densidade relativa dos indivíduos do estrato arbóreo (\%); DoR = dominância relativa dos indivíduos do estrato arbóreo (\%); FR = frequência relativa dos indivíduos do estrato arbóreo (\%); GS = grupo sucessional $(\mathrm{Pi}=$ pioneira, $\mathrm{Si}=$ secundária inicial, $\mathrm{St}=$ secundária tardia); $\mathrm{SD}=$ síndrome de dispersão $($ An $=$ anemocórica, $\mathrm{Zo}=$ zoocórica, $\mathrm{Au}=$ autocórica $)$ e $\mathrm{EV}=$ posição no estrato vertical das espécies arbóreas $(\mathrm{Sb}=$ sub-bosque, $\mathrm{In}=$ intermediário, $\mathrm{Do}=$ dossel). $\mathrm{a}=$ Não foram encontrados indivíduos no estrato regenerativo; $\mathrm{b}=$ não foram encontrados indivíduos no estrato arbóreo; c = Espécies arbóreas não classificadas de acordo com sua posição no estrato vertical, por possuírem menos de 10 indivíduos.

em relação ao regenerativo. Assim, a metodologia adotada pode não ter sido suficiente para um bom inventário da riqueza da regeneração natural. Este resultado sugere um maior esforço amostral, sobretudo para regeneração, se o objetivo do trabalho for quantificar a riqueza da floresta.

Apesar da suficiência amostral não ter sido atingida para a regeneração, a menor riqueza deste estrato em relação ao estrato arbóreo pode sugerir alguma falha na colonização de espécies presentes no estrato arbóreo, além de possíveis modificações na comunidade vegetal futura, principalmente por algumas espécies abundantes no estrato maduro da floresta, apresentarem pouco ou nenhum indivíduo dentre os regenerantes (Tabela 1). Diversos estudos apontam que a mortalidade é muito maior em juvenis e declina com a idade (GARWOOD, 1983; DE STEVEN, 1994; CONNELL e GREEN, 2000; GILBERT et al., 2001; DELISSIO; PRIMACK, 2003), logo se existem poucos indivíduos de uma determinada espécie no estrato regenerativo, aumentando-se a chance desta espécie apresentar problemas populacionais caso esses indivíduos sejam mortos nos ambientes por eventos estocásticos. No presente estudo Callisthene major Mart., Maprounea guianensis Aubl. e Schefflera morototoni (Aubl.) Maguire, Steyerm. \& Frodin, tiveram poucos indivíduos no estrato regenerativo (Tabela 1) apesar de serem comuns no estrato arbóreo, logo são espécies que devem ter suas populações estudadas em deta- 


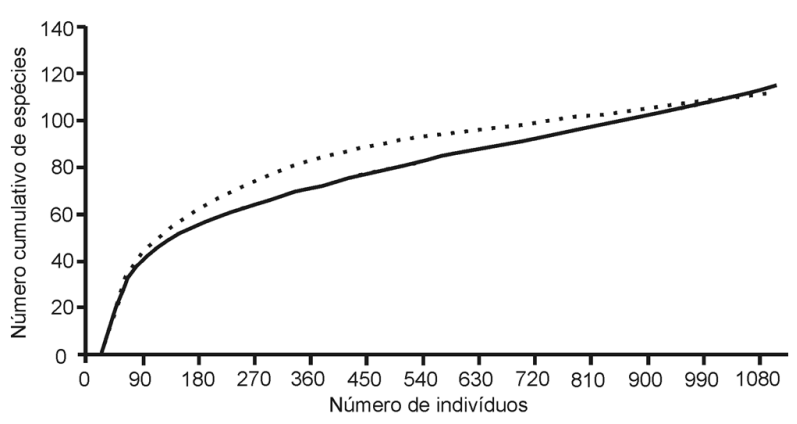

FIGURA 1: Estimador de espécies Jackknife 2 para o fragmento de Floresta Estacional Semidecidual estudada em Itumbiara, GO. Em linha pontilhada, estrato arbóreo, e em linha contígua, estrato de regeneração.

FIGURE 1: Jackknife 2 species estimator to a seasonal semideciduous forest studied in Itumbiara, Goiás. Dashed line indicates arboreal layer and the continuous line indicates the regeneration layer.

lhe para aferirmos se de fato existem problemas na regeneração.

As sete espécies com maior densidade representam $52,15 \%$ do total dos indivíduos amostrados. Por outro lado, 30 espécies tiveram apenas um ou dois indivíduos. Em algumas fitofisionomias de Florestas Tropicais é comum que cinco a dez espécies com maior densidade representem $50 \%$ do valor de importância (HARTSHORN, 1980) e o mesmo ocorre para Florestas Semideciduais preservadas no Brasil Central (DIAS NETO et al., 2009 e VALE et al., 2009). As três espécies com maior VI foram Nectandra megapotamica (Spreng.) Mez, Schefflera morototoni (Aub1.) Maguire, Steyerm. \& Frodin e Aspidosperma discolor A.DC., sendo a primeira com VI quase três vezes superior às outras duas (Tabela 1). Estas três são capazes de atingir grande porte em florestas, frequentemente ocupando o dossel da comunidade, o que explica, sobretudo, sua alta dominância relativa. Apesar de serem espécies encontradas em outros levantamentos realizados em Florestas Estacionais Semideciduais no Triângulo Mineiro (SOUZA; ARAÚJO, 2005; SOUZA et al., 2006, LOPES et al., 2011), sempre possuíam valores de importância baixos, assim como em trabalho com Florestas Estacionais Semideciduais no Estado de Goiás, onde Aspidosperma discolor, também apresentou baixo valor de importância (IMANAENCINAS et al., 2007). Assim, mesmo que não sejam incomuns em FES, raramente são as mais importantes nestas formações, este fato aumenta a importância deste fragmento para a manutenção destas espécies na região, sobretudo pelo fornecimento de sementes que podem ser dispersas para outras áreas.

Quanto às espécies pouco abundantes, Lyons et al. (2005) afirmam que espécies poucos abundantes são importantes para a ocupação de nichos vagos nas comunidades após algum processo de antropização e por ocuparem o espaço vago deixado por indivíduos mortos, assim, aumentam a riqueza da comunidade vegetal do fragmento. Essas espécies também podem desempenhar funções ecológicas semelhantes às abundantes, mas possuir tolerâncias ecológicas distintas (WALKER et al., 1999). No presente estudo, as espécies arbóreas Alibertia sessilis e Tachigali vulgaris (pioneiras), Myrcia splendens e Matayba guianensis (secundárias iniciais) e Copaifera lansdorffii (secundária tardia) foram pouco abundantes no estrato arbóreo (um ou dois indivíduos), porém, mais densas no estrato regenerativo (Tabela 1). Esse fato mostra que o trecho do fragmento florestal ainda se encontra, possivelmente, em estágio de regeneração da ação antrópica ocorrida no passado, tendo áreas com diversas condições de entrada de luz, permitindo o recrutamento de espécies de três grupos sucessionais. No fragmento florestal estudado, 29 espécies do estrato arbóreo com menos de cinco indivíduos não possuem regenerantes amostrados. Este pode ser devido ao critério de inclusão adotado (maior ou igual a $1 \mathrm{~m}$ de altura) que limitou a amostragem dos regenerantes (Figura 1), ou indicar problemas com a regeneração destas espécies.

No estrato regenerativo foram amostrados 1.116 indivíduos (4.660 ind.ha ${ }^{-1}$ ), distribuídos em 68 espécies, 40 gêneros e 26 famílias botânicas. Sete espécies representam $58,46 \%$ dos indivíduos, sendo as mais densas Cheiloclinium cognatum (Miers) A.C.Sm. e Siparuna guianensis Aubl. (Tabela 1). Estas duas espécies são características de sub-bosque, sendo consideradas secundárias tardias, e ocorrem em outras Florestas Semideciduais em médio/alto grau de conservação no Triângulo Mineiro (DIAS NETO et al., 2009; PRADO JUNIOR et al., 2010; LOPES et al., 2012). Pelo fato de espécies secundárias tardias apresentarem menor mortalidade que pioneiras no sub-bosque das florestas, por sua maior capacidade de tolerar ambientes sombreados (SWAINE et al., 1987), é esperado que muitos indivíduos dessas espécies predominem nestes locais. 
Os estratos arbóreos e regenerativos apresentaram, respectivamente, maior proporção de espécies secundárias iniciais $(61,04 \%$ e $62,32 \%)$ e secundárias tardias $(31,17$ e $28,99 \%)$, e menor proporção de pioneiras $(7,79$ e $8,70 \%$ ) (Tabela 2). Tais valores são semelhantes aos encontrados no sul de Minas Gerais (PAULA et al., 2004) e no Triângulo Mineiro (PRADO JUNIOR et al., 2010; DIAS NETO et al., 2009) para Florestas Estacionais Semideciduais em bom estágio de conservação. Considerando a alta representatividade florística das espécies secundárias iniciais, a floresta estudada parece estar em bom estágio de conservação. Isto é comprovado também pelo número de indivíduos nos grupos sucessionais (Tabela 2), cuja maior proporção é de secundárias iniciais $(78,23 \%$ para o estrato arbóreo e $67,50 \%$ para o estrato regenerativo). Esse padrão é comum para florestas em bom estágio de conservação, conforme verificado por Paula et al. (2004), Dias Neto et al. (2009) e Prado Junior et al. (2010). Outros trabalhos demonstram que a análise da proporção de número de indivíduos pode ser mais útil na classificação da floresta quanto ao seu estágio de conservação do que a proporção das espécies (GUSSON et al., 2009; DIAS NETO et al., 2009; PRADO JÚNIOR et al., 2010). No presente trabalho, o estrato arbóreo possui menor percentual de secundárias tardias $(17,11 \%)$ do que o regenerativo $(27,10 \%)$, conforme mostra a Tabela 2.

A comunidade arbórea apresentou indivíduos entre $2 \mathrm{~m}$ e $25 \mathrm{~m}$ de altura, sendo que $38,1 \%$ encontram-se entre $5 \mathrm{~m}$ a $10 \mathrm{~m}$. Três espécies fo- ram classificadas no estrato 1 (sub-bosque), dez no estrato 2 (intermediário) e seis no estrato 3 (dossel) (Tabela 1). A mediana das alturas dos indivíduos arbóreos foi $10 \mathrm{~m}$ e o terceiro quartil igual a $14 \mathrm{~m}$. Todas as espécies ocorrentes no dossel (Aspidosperma discolor, Callisthene major, Nectandra megapotamica, Ocotea corymbosa, Schefflera morototoni e Virola sebifera) são secundárias iniciais (Tabelas 1 e 3). A ausência de secundárias tardias no estrato provavelmente ocorreu por dois motivos: 1) retirada de madeira, visualizada por antigos remanescentes de desbaste e 2) o efeito deste desbaste que proporcionou sítios mais abertos, desfavorecendo espécies tolerantes à sombra a atingirem o dossel, indicando um estágio tipicamente secundário inicial no dossel. Porém, muitas espécies tolerantes à sombra não atingem o estrato superior da floresta, são espécies de sub-bosque, com característica ecológica de espécie no sub-bosque ou do estrato intermediário, onde há maior sombreamento. Estas espécies de dossel são diferentes das espécies encontradas por Lopes e colaboradores (2012) em Florestas Semideciduais do Triângulo Mineiro mostrando que o dossel de diferentes FES é distinto entre si.

As dez espécies classificadas no estrato intermediário tiveram 312 indivíduos, o que representa $27,96 \%$ do total dos indivíduos avaliados. Annona montana, Antonia ovata, Licania apetala, Maprounea guianensis, Protium heptaphyllum e Vochysia haenkeana são espécies secundárias iniciais. Emmotum nitens, Siphoneugena densiflora e

TABELA 2: Porcentagem de espécies e de indivíduos por estrato (arbóreo ou regenerativo), por grupo sucessional (GS) e por síndrome de dispersão (SD) em um fragmento de Floresta Estacional Semidecidual em Itumbiara, GO.

TABLE 2: Percentage of species and individuals in each layer (arboreal and regenerative), by sucessional group (GS) and dispersion syndrome (SD) in a semideciduous seasonal forest in Itumbiara, Goiás.

\begin{tabular}{ccccccccc}
\hline \multicolumn{9}{c}{ Arbóreo } \\
\hline $\mathrm{GS}$ & $\mathrm{NE}$ & $\%$ & $\mathrm{NI}$ & $\%$ & $\mathrm{NE}$ & $\%$ & $\mathrm{NI}$ & $\%$ \\
$\mathrm{Pi}$ & 6 & 7,79 & 52 & 4,66 & 6 & 8,70 & 252 & 5,40 \\
$\mathrm{Si}$ & 47 & 61,04 & 873 & 78,23 & 43 & 62,32 & 3148 & 67,50 \\
$\mathrm{St}$ & 24 & 31,17 & 191 & 17,11 & 20 & 28,99 & 1264 & 27,10 \\
\hline $\mathrm{SD}$ & $\mathrm{NE}$ & $\%$ & $\mathrm{NI}$ & $\%$ & $\mathrm{NE}$ & $\%$ & $\mathrm{NI}$ & $\%$ \\
\hline $\mathrm{An}$ & 21 & 27,27 & 122 & 10,93 & 14 & 20,29 & 444 & 0,10 \\
$\mathrm{Au}$ & 3 & 3,90 & 14 & 1,25 & 2 & 2,90 & 8 & 0,01 \\
$\mathrm{Zo}$ & 53 & 68,83 & 980 & 87,81 & 53 & 76,81 & 4212 & 0,89 \\
\hline
\end{tabular}

Em que: $\mathrm{NE}=$ número de espécies; $\mathrm{NI}=$ número de indivíduos por ha; $\mathrm{Pi}=$ pioneira; $\mathrm{Si}=$ secundária inicial; $\mathrm{St}=$ secundária tardia; $\mathrm{An}=$ anemocórica; $\mathrm{Zo}=$ zoocórica; $\mathrm{Au}=$ autocórica. 
Terminalia glabrescens são secundárias tardias e Xylopia aromatica, pioneira. Novamente, a maior quantidade de espécies (Tabela 1) e de indivíduos (Tabela 3) no estrato intermediário são secundários iniciais, em detrimento de tardias e pioneiras. Tanto distúrbios naturais (queda de galhos e árvores), quanto antrópicos (retirada de madeira), favorecem maior penetração de luz e proporcionam melhores condições para espécies secundárias iniciais (VALE et al., 2009).

Apenas três espécies foram classificadas como de sub-bosque: Cheiloclinium cognatum, Hirtella gracilipes e Siparuna guianensis. Duas são secundárias iniciais e uma é tardia (Tabela 1). A ausência de pioneiras (Tabela 3) indica maior sombreamento deste estrato e a mesma conclusão pode ser feita ao se comparar os grupos sucessionais do estrato arbóreo e regenerativo (Tabela 2). Há muito mais indivíduos secundários tardios no estrato regenerativo do que no arbóreo. Desta forma, o sub-bosque, possivelmente, deve apresentar sombreamento suficiente para o estabelecimento de espécies de caráter mais tardio.

Quanto à síndrome de dispersão do estrato arbóreo, 53 espécies encontradas são zoocóricas $(68,83 \%), 21$ anemocóricas $(27,27 \%)$ e três autocóricas $(3,90 \%$ ) (Tabela 2). Além disso, $87,81 \%$ dos indivíduos são zoocóricos e os demais são anemocóricos ou autocóricos. A presença de zoocoria é importante, pois em Florestas Tropicais há a produção de frutos carnosos o ano todo (SMYTHE, 1970; MORELLATO et al., 2000; MIKICHI e SILVA,

TABELA 3: Porcentagem de indivíduos de cada estrato, em uma Floresta Estacional Semidecidual no município de Itumbiara, GO.

TABLE 3: Percentage of individuals in each layer in a semideciduous seasonal forest in Itumbiara, Goiás.

\begin{tabular}{cccccccc}
\hline \multirow{2}{*}{ Estrato } & \multicolumn{3}{c}{ GS } & & \multicolumn{2}{c}{ SD } \\
\cline { 2 - 4 } \cline { 6 - 7 } & $\mathrm{Pi}$ & $\mathrm{Si}$ & $\mathrm{St}$ & & $\mathrm{An}$ & $\mathrm{Zo}$ \\
\hline Do & 0,00 & 100,00 & 0,00 & & 14,22 & 85,78 \\
$\mathrm{In}$ & 13,14 & 56,73 & 30,13 & & 8,65 & 91,35 \\
$\mathrm{Sb}$ & 0,00 & 81,59 & 18,41 & & 0,00 & 100.00 \\
\hline
\end{tabular}

Em que: (Do = dossel; In = intermediário; $\mathrm{Sb}=$ subbosque) pertencentes a cada grupo sucessional (GS) e a cada síndrome de dispersão (SD) em um fragmento de Floresta Estacional Semidecidual em Itumbiara, GO. Pi $=$ pioneira; $\mathrm{Si}=$ secundária inicial; $\mathrm{St}=$ secundária tardia; $\mathrm{An}=$ anemocórica; $\mathrm{Zo}=$ zoocórica) .
2000) e os fragmentos florestais existentes servem de refúgio constante para fauna do Cerrado, cuja produção de frutos carnosos é severamente reduzida na estação seca (OLIVEIRA e PAULA, 2001). Resultados semelhantes foram encontrados em outras Florestas Estacionais estudadas por Nunes et al. (2003), no Sul de Minas Gerais e por Santos e Kinoshita (2003) e Yamamoto et al. (2007) no Estado de São Paulo.

Quanto à síndrome de dispersão na estratificação vertical, a zoocoria foi mais presente em espécies de sub-bosque (Tabela 3 ). O contrário é perceptível para anemocóricas, cuja maior representatividade foi nas espécies de dossel (Tabela 3). Como as árvores do estrato superior da floresta estão mais sujeitas ao vento, espécies anemocóricas apresentam mais indivíduos no dossel, pois há melhores chances de dispersão (YAMAMOTO et al., 2007; SPINA et al., 2001).

A investigação das síndromes de dispersão ligadas à estratificação é relevante, pois a fauna pode ocupar distintos estratos na comunidade vegetal. Estudos demonstram que existem animais dependentes do dossel, como grandes aves, e outros dependentes do sub-bosque, como pequenos mamíferos e pequenas aves granívoras (PEARSON, 1971; CLARK e POULSEN, 2001).

\section{CONCLUSÕES}

O trabalho buscou detalhar a estrutura horizontal e vertical do fragmento estudado, com o qual foi possível classificar as espécies quanto ao seu estrato dominante e melhor descrever a comunidade pela caracterização do estrato regenerativo. Acredita-se que a análise de regeneração e comunidade arbórea seja importante para realização de previsões sobre o possível futuro das comunidades. Além disso, demonstrou-se a importância desta floresta no fornecimento de recursos para a fauna, com a produção de frutos carnosos dispersos por animais. Além disso, apesar da estrutura do trecho da floresta indicar corte seletivo de madeira, a mesma é importante para a manutenção da diversidade como um todo, pois possui alta densidade de diversas espécies pouco representadas em outras áreas da região e das próprias Florestas Estacionais Semideciduais. Ressalta-se a importância da conservação deste fragmento e de outros na região que é dominada por plantações de cana-de-açúcar, visando à manutenção da flora e fauna associadas a esses ambientes florestais. 


\section{REFERÊNCIAS BIBLIOGRÁFICAS}

ARAÚJO, G. M.; HARIDASAN, M. Estrutura fitossociológica de duas matas mesófilas semidecíduas, em Uberlândia, Triângulo Mineiro. Naturalia, Rio Claro, v. 22, p. 115-129, 1997.

CATHARINO, E. L. M. et al. Aspectos da composição e diversidade do componente arbóreo das florestas da Reserva Florestal do Morro Grande, Cotia, SP. Biota Neotropica, Campinas, v. 6, p. 1-28, 2006.

CECCON, E.; HUANTE, P.; RINCÓN, E. Abiotic Factors Influencing Tropical Dry Forests Regeneration. Brazilian Archives of Biology and Technology, Curitiba, v. 49, n. 2, p. 305-312, 2006. APG III. A phylogenetic classification of land plants to accompany APG III. Botanical Journal of the Linnean Society v. 161, n. 2, p. 122-127, 2009.

CLARK, C. J.; POULSEN, J. R. The role of arboreal seed dispersal groups on the seed rain of a lowland tropical forest. Biotropica, v. 33, p. 606-620, 2001. CONNELL, J. H.; GREEN, P. T. Seedling dynamics over thirty-two years in tropical rain forest tree. Ecology, v. 81, n. 2, p. 568-584, 2000.

COLWELL, R. K. 2005. EstimateS: Statistical estimation of species richness and shared species form samples. Versão 7.5.

COLWELL, R. K.; CODDINGTON, J. A. Estimating the extent of terrestrial biodiversity through extrapolation. Philosophical Transactions of the Royal Society of London. Series B, Biological Sciences, v.345, p. 101-118, 1994.

DE STEVEN, D. Tropical Tree Seedling Dynamics: Recruitment Patterns and Their Population Consequences for Three Canopy Species in Panama. Journal of Tropical Ecology, v. 10, n.3, p. 369383, 1994.

DELISSIO, L. J.; PRIMACK, R. B. The impact of drought on the population dynamics of canopy-tree seedlings in an aseasonal Malaysian rain forest. Journal of Tropical Ecology v. 19, p. 489-500, 2003.

DIAS NETO, O. C. et al. Estrutura fitossociológica e grupos ecológicos em fragmento de Floresta Estacional Semidecidual, Uberaba, Minas Gerais, Brasil. Rodriguésia, Rio de Janeiro, v. 60, n. 4, p. 1087-1100, 2009.

FERREIRA JÚNIOR,W. G. et al. Composição florística da vegetação de um trecho de Floresta Estacional Semidecidual em Viçosa, Minas Gerais, e espécies de maior ocorrência na região. Revista Árvore, Viçosa, v. 31, n. 6, p. 1121-1130, 2007.
GANDOLFI, S.; LEITÃO-FILHO, H. F.; BEZERRA, C. L. Levantamento florístico e caráter sucessional das espécies arbustivo-arbóreas de uma Floresta Mesófila Semidecídua no município de Guarulhos, SP. Revista Brasileira de Biologia, São Carlos, v. 55, p. 752-767, 1995.

GARWOOD, N. C. Seed germination in a seasonal tropical forest in Panama: a community study. Ecology Monographs, v. 53, p. 159-181, 1983.

GILBERT, I. R.; JARVIS, P. G.; SMITH, H. Proximity signal and shade avoidance differences between early and late successional trees. Nature, v. 411, p. 792-795, 2001.

GUSSON, A. E. et al. Características químicas do solo e estrutura de um fragmento de Estacional Semidecidual em Ipiaçu, Minas Gerais, Brasil. Rodriguésia, Rio de Janeiro, v. 60, p. 403-414, 2009.

HARTSHORN, G. S. Neotropical Forest Dynamics. Biotropica, v. 12, p. 23-30, 1980.

IBGE. Mapa de Solos do Brasil. Rio de Janeiro. 2001.

IMAÑA-ENCINAS, J. et al. Estrutura diamétrica de um trecho de floresta estacional semidecidual na área do ecomuseu do cerrado, Goiás. Cerne, Lavras, v. 15, n. 2, p. 155-165, 2009

INOSHITA, L. S. et al. Composição florística e síndromes de polinização e de dispersão da mata do Sítio São Francisco, Campinas, SP, Brasil. Acta Botanica Brasilica, Novo Horizonte, v. 20, p. 313327, 2006.

KÖPPEN, W. Climatologia: con un estudio de los climas de la tierra. México: Fondo de Cultura Econômica, 1948.

KREBS, C. J. Ecological Methodology. New York: Wesley Longman, 2000.

LOPES, S. F. et al. An Ecological Comparison of Floristic Composition in Seasonal Semideciduous Forest in Southeast Brazil: Implications for Conservation. International Journal of Forestry Research v. 2012, p. 1-14, 2012.

LYONS, K. G. et al. Rare Species and Ecosystem Functioning. Conservation Biology, v.19, n. 4, p. 1019-1024. 2005.

MACHADO, E. L. M. et al. Análise comparativa da estrutura e flora do compartimento arbóreoarbustivo de um remanescente florestal na Fazenda Beira Lago, Lavras, MG. Revista Árvore, Viçosa, v. 28, p. 499-516, 2004.

MARANGON, L. C. et al. Regeneração natural em um fragmento de Floresta Estacional Semidecidual em Viçosa, Minas Gerais. Revista Árvore, Viçosa, 
V. 32, p. 183-191, 2008.

MARCHIORI, C. H. et al. Parasitóides da Subfamília Eucoilinae (Hymenoptera: Figitidae) coletados em armadilhas de bacias amarelas e armadilhas Malaise em Araporã, Minas Gerais e Itumbiara, Goiás. Arquivos do Instituto Biologico, São Paulo, v. 70, p. 207-209, 2003.

MIKICHI, S. B.; SILVA, S. M. Composição florística e fenologia das espécies zoocóricas de remanascentes de Floresta Estacional Semidecidual no Centro-oeste do Paraná, Brasil. Acta Botanica Brasílica, Novo Horizonte, v. 15, v. 1, p. 89-113, 2000.

MORELlato, L. P. C. et al. Phenology of Atlantic Rain Forest Trees: A Comparative Study.

Biotropica, v. 32, n. 4b, p. 811-823, 2000.

MUELLER-DOMBOIS, D.; ELLENBERG, $\mathrm{H}$. Aims and methods of vegetation ecology. New York: Wiley \& Sons. 1974.

MYERS, N. et al. Biodiversity hotspots for conservation priorities. Nature, v. 403, p. 853-858, 2000.

NUNES, Y. R. F. et al. Variações da fisionomia, diversidade e composição de guildas da comunidade arbórea em um fragmento de Floresta Semidecidual em Lavras, MG. Acta Botanica Brasilica, Novo Horizonte, v. 17, p. 213-229, 2003.

OLIVEIRA, P. E. A. M.; PAULA, F. R. Fenologia e biologia reprodutiva de plantas de Matas de Galeria. In: Cerrado: caracterização e recuperação de Matas de Galeria. RIBEIRO, J. F. ; FONSECA, C. A. L. ; SOUSA-SILVA, J. C. (eds.). Planaltina, EMBRAPA Cerrados, 2001. p. 303-328.

OLIVEIRA-FILHO, A. T. et al. Comparison of the woody flora and soils of six areas of Montane Semideciduous Forest in Southern Minas Gerais, Brazil. Edinburgh Journal Botany, v. 51, n. 3, p. 355-389, 1994.

OLIVEIRA-FILHO, A. T.; FONTES, M. A. Patterns of floristic differentiation among atlantic forests in Southern Brazil and the influence of climate. Biotropica, v. 32, n. 4b, p. 793-810, 2000.

OLIVEIRA-FILHO, A. T.; MELLO, J. M.; SCOLFORO, J. R. S. Effects of past disturbance and edges on tree community structure and dynamics within a fragmento of tropical semideciduous forest in south-easten Brazil over five-year period (19871992). Plant Ecology, v. 131, p. 45-66, 1997.

PARE, S. et al. Regeneration and spatial distribution of seedling populations in Sudanian dry forests in relation to conservation status and human pressure. Tropical Ecology, v. 50, n. 2, p. 339-353, 2009.
PAULA, A. D. et al. Sucessão ecológica da vegetação arbórea em uma Floresta Estacional Semidecidual, Viçosa, MG, Brasil. Acta Botanica Brasilica, Novo Horizonte, v. 18, p. 407-423, 2004. PEARSON, D. L. Vertical stratifcation of birds in a tropical dry forest. The Condor, v. 73, p. 46-55, 1971.

PIVELLO, V. R. et al. Chuva de sementes em fragmentos de Floresta Atlântica (São Paulo, SP, Brasil), sob diferentes situações de conectividade, estrutura florestal e proximidade da borda. Acta Botanica Brasilica, Novo Horizonte, v. 20, p. 845859, 2006.

PRADO JÚNIOR, J. A. et al. Estrutura da comunidade arbórea em um fragmento de Floresta Estacional Semidecidual localizada na reserva legal da fazenda Irara, Uberlândia, MG. Bioscience Journal, Uberlândia, v. 26, n. 4, p. 683-647, 2010. RIZZINI, C. T. Tratado de fitogeografia do Brasil. Âmbito Cultural, 1997.

SALLES, J.C.; SCHIAVINI, I. Estrutura e composição do estrato de regeneração em um fragmento florestal urbano: implicações para a dinâmica e a conservação da comunidade arbórea. Acta Botanica Brasilica, Novo Horizonte, v. 21, p. 223-233, 2007.

SANTOS, K. D.; KINOSHITA, L. S. Flora arbustivoarbórea do fragmento de Floresta Estacional Semidecidual do Ribeirão Cachoeira, município de Campinas, SP. Acta Botanica Brasilica, Novo Horizonte, v. 17, p. 325-341, 2003.

SCHAAF, T. The Future of Dry Lands. Paris: UNESCO, 2006.

SHEPHERD, G. J. Fitopac Shell 1.6.4.29. Campinas: Depto. de Botânica. UNICAMP, 2006.

SILVA, N. R. S. et al. Composição florística e estrutura de uma Floresta Estacional Semidecidual montana em Viçosa, MG. Revista Árvore, Viçosa, v. 28, p. 397-405, 2004.

SMYTHE, N. Relationships between Fruiting Seasons and Seed Dispersal Methods in a Neotropical Forest. The American Naturalist, v. 104, n. 935 , p. 25-35, 1970.

SOUZA, J. P. D. et al. Comparison between canopy trees and arboreal lower strata of urban semideciduous seasonal forest in Araguari, MG. Brazilian Archives of Biology and Technology, Curitiba, v. 49, p. 775-783, 2006.

SPINA, A. P.; FERREIRA, W. N.; LEITÃO-FILHO, H. F. Floração, frutificação e síndromes de dispersão de uma comunidade de Floresta de Brejo na região de Campinas (SP). Acta Botanica Brasilica, Novo 
Horizonte, v. 15, n. 3, p. 349-368, 2001.

SWAINE, M. D. ; WHITMORE, T. C. On the definition of ecological species groups in tropical rain forests. Vegetatio, v. 75, p. 81-86, 1988.

SWAINE, M. D.; LIEBERMAN, D.; PUTZ, F. E. The dynamics of tree populations in tropical forest: a review. Journal of Tropical Ecology, v. 3, p. 359:366, 1987.

TABARELLI, M.; MANTOVANI, W.; PERES, C. A. Effects of habitat fragmentation on plant guild structure in the montane Atlantic forest of southeastern Brazil. Biological Conservation, v. 91, p. 119-127, 1999.

TONIATO, M. T. Z.; OLIVEIRA-FILHO, A. T. Variations in tree community composition and structure in a fragment of tropical semideciduous forest in southeastern Brazil related to different human disturbance histories. Forest Ecology and Management, v. 198, p. 319-339, 2004.

TURNER, I. M. Species lossin fragments of tropical rainforest: a review of the evidence. Journal of Applied Ecology, v. 33, p. 200-209, 1996.

VALE, V.S. et al. Composiçãoflorística e estrutura do componente arbóreo em um remanescente primário de Floresta Estacional Semidecidual em Araguari, Minas Gerais, Brasil. Hoehnea, Água Funda, v. 36, p. 417-429, 2009.

van der PIJL, L. Principles of dispersal in higher plants. New York: Springer Verlag. 1982. VELOSO, P. H.; RANGEL FILHO, A. L. R.; LIMA, J. C. A. Classificação da vegetação brasileira adaptada a um sistema universal. IBGE. 1991. VIEIRA, D. L. M.; SCARIOT, A. Principles of natural regeneration of tropical dry forests for restoration. Restoration Ecology, v. 14, p. 11-20, 2006.

WALKER, B.; KINZIG, A.; LANGRIDGE, J. Plant Attribute Diversity, Resilience, and Ecosystem Function: The Nature and Significance of Dominant and Minor Species. Ecosystems, v. 2, p. 95-113, 1999.

YAMAMOTO, L. F.; KINOSHITA, L. S.; MARTINS, F. R. Florística dos componentes arbóreo e arbustivo de um trecho da Floresta Estacional Semidecídua Montana, município de Pedreira, estado de São Paulo. Revista Brasileira de Botanica, São Paulo, v. 28, p. 191-202, 2005.

YAMAMOTO, L. F.; KINOSHITA, L. S.; MARTINS, F. R. Síndromes de polinização e de dispersão em fragmentos da Floresta Estacional Semidecídua Montana, SP, Brasil. Acta Botanica Brasilica, Novo Horizonte, v. 21, p. 553-573, 2007. ZIPPARRO, V. B. et al. Levantamento florístico de Floresta Atlântica no sul do Estado de São Paulo, Parque Estadual Intervales, Base Saibadela. Biota Neotropica, v. 5, p. 127-144, 2005. 